\title{
Variability of in vivo reference point indentation in skeletally mature inbred rats
}

\author{
Matthew R. Allen ${ }^{1}$, Christopher L. Newman ${ }^{1}$, Eric Smith ${ }^{1}$, Drew M. Brown ${ }^{1}$, and Jason M. \\ Organ ${ }^{1}$ \\ ${ }^{1}$ Department of Anatomy and Cell Biology, Indiana University School of Medicine, Indianapolis, \\ IN, United States.
}

\begin{abstract}
Reference point indentation (RPI) has emerged as a novel tool to measure material-level biomechanical properties in vivo. Human studies have been able to differentiate fracture versus non-fracture patients while a dog study has shown the technique can differentiate drug treatment effects. The goal of this study was to extend this technology to the in vivo measurement of rats, one of the most common animal models used to study bone, with assessment of intra- and interanimal variability. Seventy-two skeletally mature male Sprague-Dawley rats were subjected to in vivo RPI on the region between the tibial diaphysis and proximal metaphysis. RPI data were assessed using a custom MATLAB program to determine several outcome parameters, including first cycle indentation distance (ID- $1^{\text {st }}$ ), indentation distance increase (IDI), total indentation distance (TID), first cycle unloading slope (US-1 ${ }^{\text {st }}$ ), and first cycle energy dissipation (ED-1 ${ }^{\text {st }}$ ). Intra-animal variability ranged from $13-21 \%$ with US- $1^{\text {st }}$ and Tot Ed $1^{\text {st }}-\mathrm{L}$ being the least variable properties and IDI the most highly variable. Inter-animal variability ranged from $16 \%$ (US- $1^{\text {st }}$ ) to $25 \%$ (ED- $1^{\text {st }}$ and IDI). Based on these data, group size estimates would need to range from 9-18/ group to achieve sufficient power for detecting a $25 \%$ difference in a two-group experiment. Repeat tests on the contralateral limb of a small cohort of animals $(n=17)$ showed non-significant differences over 28 days ranging from $-6 \%$ to $-18 \%$. These results provide important data on RPI variability (intra- and inter-animal) in rats that can be used to properly power future experiments using this technique.
\end{abstract}

\section{Keywords}

bone material properties; bone mechanics; microindentation

C 2014 Elsevier Ltd. All rights reserved.

Send Correspondence to: Matthew R. Allen, PhD, Dept. of Anatomy and Cell Biology, MS 5035, Indiana University School of Medicine, 635 Barnhill Dr., Indianapolis, IN 46202, Tel: 317-274-1283, FAX: 317-278-2040, matallen@iupui.edu.

Publisher's Disclaimer: This is a PDF file of an unedited manuscript that has been accepted for publication. As a service to our customers we are providing this early version of the manuscript. The manuscript will undergo copyediting, typesetting, and review of the resulting proof before it is published in its final citable form. Please note that during the production process errors may be discovered which could affect the content, and all legal disclaimers that apply to the journal pertain.

Confilict of Interest statement

The authors have no conflict of interest related to this work. 


\section{INTRODUCTION}

Assessment of biomechanical properties has long been confined to pre-clinical studies and, more specifically, ex-vivo mechanical tests. Recent technology, termed reference point indentation (RPI), has made it possible to assess biomechanical properties in vivo (Hansma et al., 2008). In vivo studies have shown that RPI can differentiate between patients who have fractured versus non-fracture patients (Diez-Perez et al., 2010) as well as patients who have been treated with bisphosphonates versus those who were treatment naïve (GüerriFernández et al., 2012). In vivo testing of dogs has shown RPI can differentiate raloxifene treatment from controls after six months of clinically relevant dosing (Aref et al., 2013). In addition, a related device (Osteoprobe) that operates using slightly different technology revealed significant differences in the material properties of patients with diabetes versus healthy controls (Farr et al., 2014). Collectively, these data show promise for RPI technology to allow minimally invasive measures of material-level biomechanical properties.

Rodents represent the most commonly used animal model to study bone and are often the model first used to evaluate novel interventions (Kalu, 1991; Thompson et al., 1995).

Although several studies have assessed biomechanical properties of rodent bone ex vivo, there have been no reports of in vivo assessment of rodents. The goal of this study was to determine the intra- and inter-animal variability, as well as the variability over time (in order to understand potential variability that might occur in control animals in future intervention studies), for in vivo measures with RPI in skeletally mature rats. These data will be essential to understand the practicality of the technique for use in rats as well as to provide variability data to help design adequately powered experiments.

\section{METHODS}

\section{Experimental design}

Seventy-two skeletally mature male (6 month old) Sprague Dawley rats were purchased (Harlan) and acclimatized for one week prior to reference point indentation (RPI) testing. A subset of animals $(\mathrm{n}=17)$ underwent a second RPI test session 28 days after the first test. These repeat test sessions were performed on the contralateral limb to avoid any local tissue damage caused by the first test session. Following each testing session, animals were returned to their cages. These animals were part of a larger experiment that is outside the scope of this current report. All procedures were approved by the Indiana University School of Medicine Animal Care and Use Committee prior to the start of the study.

\section{Reference point indentation (RPI)}

Material-level mechanical properties of the anterior surface of the tibial cortex were assessed in vivo using RPI (Biodent Hfc, Active Life Scientific, Santa Barbara, CA). This site was chosen as it has been utilized previously in human and dog in vivo studies, and its limited soft tissue coverage facilitates easy access to the bone surface. The cortical thickness in this region is around $4 \mathrm{~mm}$ thick. Rats were placed under general anesthesia using inhalation isoflurane, and a local anesthetic was injected just beneath the skin in the region 
of testing. Skin overlying the region was pierced with a sterile BP1 probe contained within the measurement head unit (MHU) attached to a modified holder apparatus (Figure 1). The MHU was lowered vertically, normal to the surface of the bone, until the probe assembly rested on the bone surface. As opposed to previous in vivo work in humans and dogs, we did not scrape the periosteum prior to testing due to challenges working in the small target area. Following positioning of the reference probe, a reference force of $\sim 13$ Newtons was applied to stabilize the MHU, and the measurement protocol was initiated. Measurements began with a series of four preconditioning cycles ( $1 \mathrm{~N}$ force at $5 \mathrm{~Hz}$ ) followed by a series of 10 testing cycles $(10 \mathrm{~N}$ at $2 \mathrm{~Hz})$. This force was chosen to match in vivo levels used previously in humans and dogs. To achieve our goal of three usable tests for each animal, between three and seven measurements, within a few mm of each other, were collected. For the multiple tests on each animal an average was taken for a given parameter and that data-point was used to compare that parameter across animals. All animals were conscious and mobile $\sim 10$ minutes post-testing. There was no sign of post-test pain or discomfort as assessed by visual inspection of animals during normal cage activity.

Raw data output from the RPI analysis software (version 2.0) were imported into a customized MATLAB code (Mathworks) (Aref et al., 2013). Primary variables of interest from the MATLAB program include first cycle indentation distance (ID- ${ }^{\text {st }}$ ), which represents the depth the probe penetrated on the initial cycle; first cycle energy dissipation $\left(\mathrm{ED}-\mathrm{1}^{\mathrm{st}}\right)$, which represents the energy dissipated in the first cycle; first cycle unloading slope (US-1 ${ }^{\text {st }}$ ) which represents material stiffness (damage modulus) for the first cycle; indentation distance increase (IDI), which represents the penetration depth between the first and $10^{\text {th }}$ cycle; total indentation distance (TID),) which represents the distance from the bone surface to the depth of penetration after the $10^{\text {th }}$ cycle; and total energy dissipation (Tot ED) which represents the total energy dissipation summed over all 10 cycles (Figure 1). Our previous work has shown that parameters analyzed by the MATLAB software that were also generated by the manufacturer software yielded correlation coefficients of $>0.96$ (Aref). The advantage of the MATLAB program over the manufacturer software is that additional data, specifically cycle-by-cycle and energy data are generated.

\section{Data Analyses}

Intra-animal variability was assessed by calculating the coefficient of variation (CV) for all tests within an animal. Inter-animal variation was assessed by calculating CVs for each outcome parameter across all animals. Paired t-test analyses were used to compare baseline and 28 day data.

\section{RESULTS}

A total of 319 tests were conducted in the 72 animals. Of these, 49 tests were deemed unsuccessful during testing based on the operators noting various problems with the tests. These included the test having a negative IDI (probe final position is above original reference position), decreasing displacement in first few cycles (resulting in a negative loading slope), or the measurement unit shifting during test. Upon removal of these 
unsuccessful tests, 71 animals had between 2 and 5 measures, and these were used for subsequent analyses (one animal was removed because it had only one acceptable measure).

Intra-animal variation of RPI parameters ranged from between 13.3 and 20.6\% (Table 1, Figure 2A). The least variable parameters within animals were US $1^{\text {st }}$ and Tot ED $1^{\text {st }}$ L, each with a coefficient of variation of $13 \%$. The most variable parameter within animals was IDI a CV of $20.6 \%$. Inter-animal variation ranged from 16-25\% (Table 2, Figure 2B). The least variable parameter among animals was US- $1^{\text {st }}(\mathrm{CV}=16 \%)$, while both ED- $1^{\text {st }}$ and IDI had the largest CVs of $25 \%$.

One month following the initial RPI tests, a subset of animals $(n=17)$ underwent a second RPI test on the contralateral limb. In this smaller dataset, intra-animal variation ranged from 45-74\% with TID and ID-1st being the least variable parameters and US-1 ${ }^{\text {st }}$ the most variable. The inter-animal variability in this data set ranged from 13-23\% with the least variable parameter being US- $1^{\text {st }}$ and the most variable being TID and ID- ${ }^{\text {st }}$ (data not shown).

Changes between baseline and one month measures were calculated to determine variability over time in untreated animals. All six parameters were, on average, lower at the second measurement relative to the first, with decreases ranging from $-6 \%$ to $-18 \%$ (Table 3). For each parameter, there was a wide range of responses with some animals increasing, some decreasing, and others unchanged (Figure 3). There was no significant difference in any parameter between baseline and day 28 .

\section{DISCUSSION}

There is significant experimental value in assessing outcome variables in vivo yet from the perspective of biomechanical properties this presents unique challenges. Serum/urine biomarkers have long been used to track bone remodeling parameters over time, and recent advances in imaging have allowed for high resolution in vivo longitudinal measures of bone density and structure (Bouxsein and Delmas, 2008). These measures allow individual variability to be accounted for in statistical analyses, allowing for the utilization of fewer subjects/animals compared to traditional cross-sectional designs. The development of reference point indentation (RPI) technology has made it possible now to assess materiallevel biomechanical properties of bone in vivo (Aref et al., 2013; Diez-Perez et al., 2010). Although studies have used RPI in rodent bone ex vivo (Gallant et al., 2013), no data exists on its in vivo application in this animal model.

Inter-individual variability of in vivo measures on human patients has been reported to be between 15\% and 24\% for IDI and 10-17\% for Total ID (Diez-Perez et al., 2010; GüerriFernández et al., 2012). Inter-individual variability of in vivo measurements in dogs ranged from 5\% (US-1 ${ }^{\text {st }}$ ) to 27\% (ID-1 ${ }^{\text {st }}$ and Energy-1 ${ }^{\text {st }}$ ) (Aref et al., 2013). Our current work in rats falls within these same ranges. Because of the larger data set $(n=71)$ and use of inbred rats, interindividual variability should be lower than previous studies in dogs and humans. One potential explanation for this is size differences. The test is being conducted over a larger percentage of the total bone length in rats compared to dogs and humans and thus may 
be incorporating more of the natural variability in properties that exist along the length of the bone. The small size also presents challenges to orienting the test set-up that are not of concern in larger test subjects. Alternatively, the lack of periosteum scraping in the rats may increase the variation. Due to the small target region we opted not to scrape periosteum as has been done in dogs and humans. It is also possible that the inherent properties of the microstructure in rat bone are simply more variable than they are in dogs or humans.

In an attempt to put the inter-animal variations into context, we calculated CVs for an archived set of untreated rat femoral whole bone three-point bending tests from our laboratory (Table 4). These values range from 7-30\% for common parameters such as ultimate load, stiffness, and energy to failure, suggesting that RPI tests produce data that fall near the upper range of variability produced by traditional ex vivo mechanical tests. Despite its variability, though, RPI is currently the only technique that provides in vivo measurements of skeletal material properties in rodents. This is valuable as it would allow for the reduction of animal numbers and, if the effect sizes are sufficient, the detection of changes in mechanical properties over time. For example, in an experimental design of two groups, the number of animals needed to detect, with $80 \%$ power, a $25 \%$ difference in outcomes based on the inter-animal variation the study would need between 9-18 animals per group at any single time point of measure (Table 2).

The presented data should be considered within the context of some limitations. As this was the first attempt to extend this in vivo technology to rats, refinement in this technique could lower the variation in future studies. Despite our previous experience with in vivo testing (Aref et al., 2013), the smaller length scale of the rat, relative to the dog, was challenging. The development of hardware to help standardize position of test locations along the length of the tibia may help reduce intra-individual variability. We also conducted repeated measures on the contralateral limb without knowledge of side-to-side variability in RPI properties. Therefore, we are unable to determine whether decreases in the values of RPI parameters between time points is a product of time (and/or growth) or a product of variability between limbs. Our rationale for not performing repeat tests on the same limb was based on the assumption there would be residual damage (or healing in response to damage) at the 28-day time point. Hence, we aimed to avoid any influence of such damage on the second measure. Whether or not such damage persists remains unknown and should be the focus of future work as testing of the same limb is likely to reduce variability. Previous work on ex vivo specimens has shown that lower load values (5N versus $10 \mathrm{~N}$ ) yielded lower viability (Setters and Jasiuk, 2014) thus it's possible that using lower loads in vivo would have benefit.

In conclusion, we present data on the in vivo variability of reference point indentation testing in skeletally mature rats. These data will provide a foundation for designing future studies using this technology by providing the intra-, inter-, and repeated measure variability in measures. 


\section{Acknowledgments}

Funding for this study was provided by NIH (AR 62002 and DK100093) and the Indiana Clinical and Translational Science Institute fellowship program. The authors would like to thank Joey Wallace and David Burr for helpful comments on early drafts of this manuscript.

\section{REFERENCES}

Aref M, Gallant MA, Organ JM, Wallace JM. ... >In vivo reference point indentation reveals positive effects of raloxifene on mechanical properties following 6months of treatment in skeletally mature beagle dogs. Bone. 2013; 56:449-453. [PubMed: 23871851]

Bouxsein ML, Delmas PD. Considerations for Development of Surrogate Endpoints for Antifracture Efficacy of New Treatments in Osteoporosis: A Perspective. J Bone Miner Res. 2008; 23:11551167. [PubMed: 18318643]

Diez-Perez A, Güerri R, Nogues X, Cáceres E, Peña MJ, Mellibovsky L, Randall C, Bridges D, Weaver JC, Proctor A, Brimer D, Koester KJ, Ritchie RO, Hansma PK. Microindentation for in vivo measurement of bone tissue mechanical properties in humans. J Bone Miner Res. 2010; 25:1877-1885. [PubMed: 20200991]

Farr JN, Drake MT, Amin S, Melton LJ, McCready LK, Khosla S. In vivo assessment of bone quality in postmenopausal women with type 2 diabetes. J Bone Miner Res. 2014; 29:787-795. [PubMed: 24123088]

Gallant MA, Brown DM, Organ JM, Allen MR, Burr DB. Reference-point indentation correlates with bone toughness assessed using whole-bone traditional mechanical testing. Bone. 2013; 53:301-305. [PubMed: 23274349]

Güerri-Fernández RC, Nogués X, Quesada Gómez JM, Torres del Pliego E, Puig L, García-Giralt N, Yoskovitz G, Mellibovsky L, Hansma PK, Diez-Perez A. Microindentation for in vivo measurement of bone tissue material properties in atypical femoral fracture patients and controls. J Bone Miner Res. 2012; 28:162-168. [PubMed: 22887720]

Hansma P, Turner P, Drake B, Yurtsev E, Proctor A, Mathews P, Lelujian J, Randall C, Adams J, Jungmann R, Garza-De-Leon F, Fantner G, Mkrtchyan H, Pontin M, Weaver A, Brown MB, Sahar N, Rossello R, Kohn D. The bone diagnostic instrument II: Indentation distance increase. Rev. Sci. Instrum. 2008; 79:064303-064303-8. [PubMed: 18601422]

Kalu D. The ovariectomized rat model of postmenopausal bone loss. Bone and mineral. 1991; 15:175191. [PubMed: 1773131]

Setters A, Jasiuk I. Towards a standardized reference point indentation testing procedure. Journal of the Mechanical Behavior of Biomedical Materials. 2014; 34:57-65. [PubMed: 24556325]

Thompson DD, Simmons HA, Pirie CM, Ke HZ. FDA Guidelines and animal models for osteoporosis. Bone. 1995; 17:125S-133S. [PubMed: 8579908] 


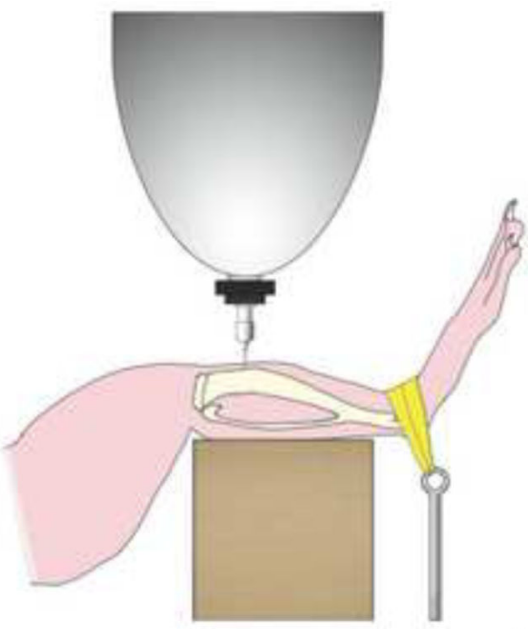

\section{First cycle}

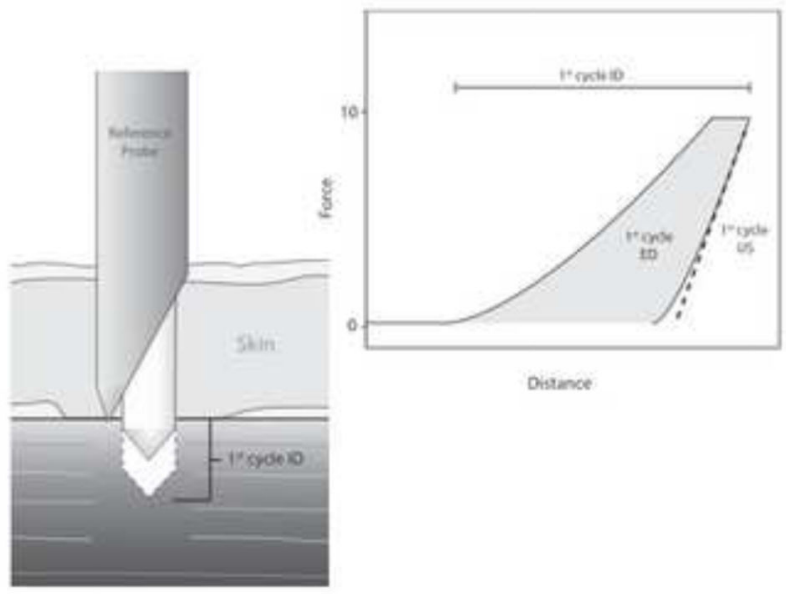

10th cycle

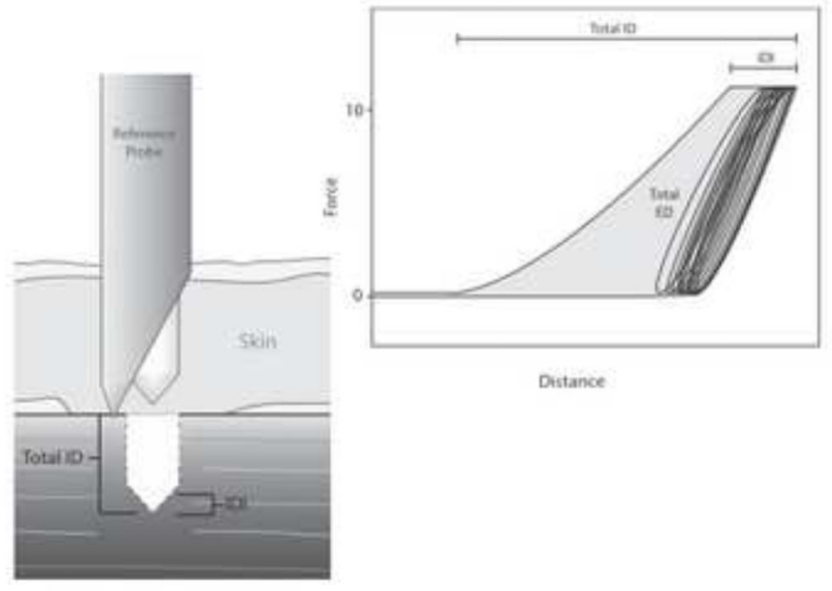

Figure 1.

In vivo testing set up and outcome parameters for RPI in skeletally mature rats. (A) The animals lower limb was flexed at the knee joint and placed on an elevated support so that the proximal tibial plateau was perpendicular to the testing probe. The foot was secured in place at the ankle and then a series of 10 cyclic indents were initiated where the test probe penetrates to a force of $10 \mathrm{~N}$ and then retracts. (B) Following the first cycle of the cyclic test, key outcomes of $1^{\text {st }}$ cycle indentation distance ( $1^{\text {st }}$ cycle ID), $1^{\text {st }}$ cycle unloading slope ( $1^{\text {st }}$ cycle US) and $1^{\text {st }}$ cycle energy dissipation $\left(1^{\text {st }}\right.$ cycle ED) can be calculated. Additional parmeters are obtained after the $10^{\text {th }}$ cycle, including total indentation distance (Total ID), indentation distance increase (IDI) and energy dissipation (Total ED). 

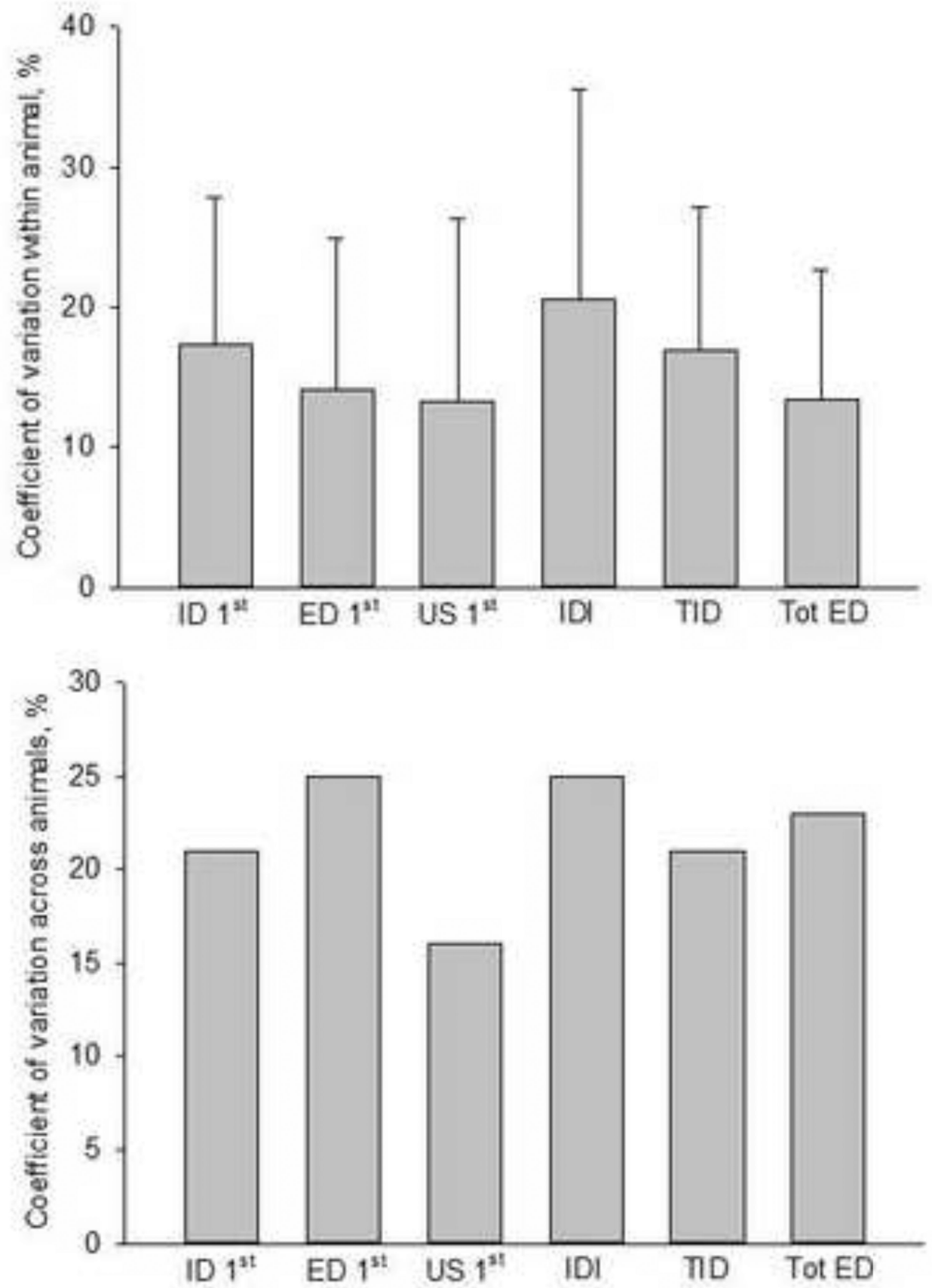

Figure 2.

RPI variability within animal and among animals. (A) Intra-animal variation, presented as the mean and standard deviation of the coefficient of variation (\%) within a given animal. (B) Inter-animal variation, presented as the CV (\%) for each variable across all animals. 


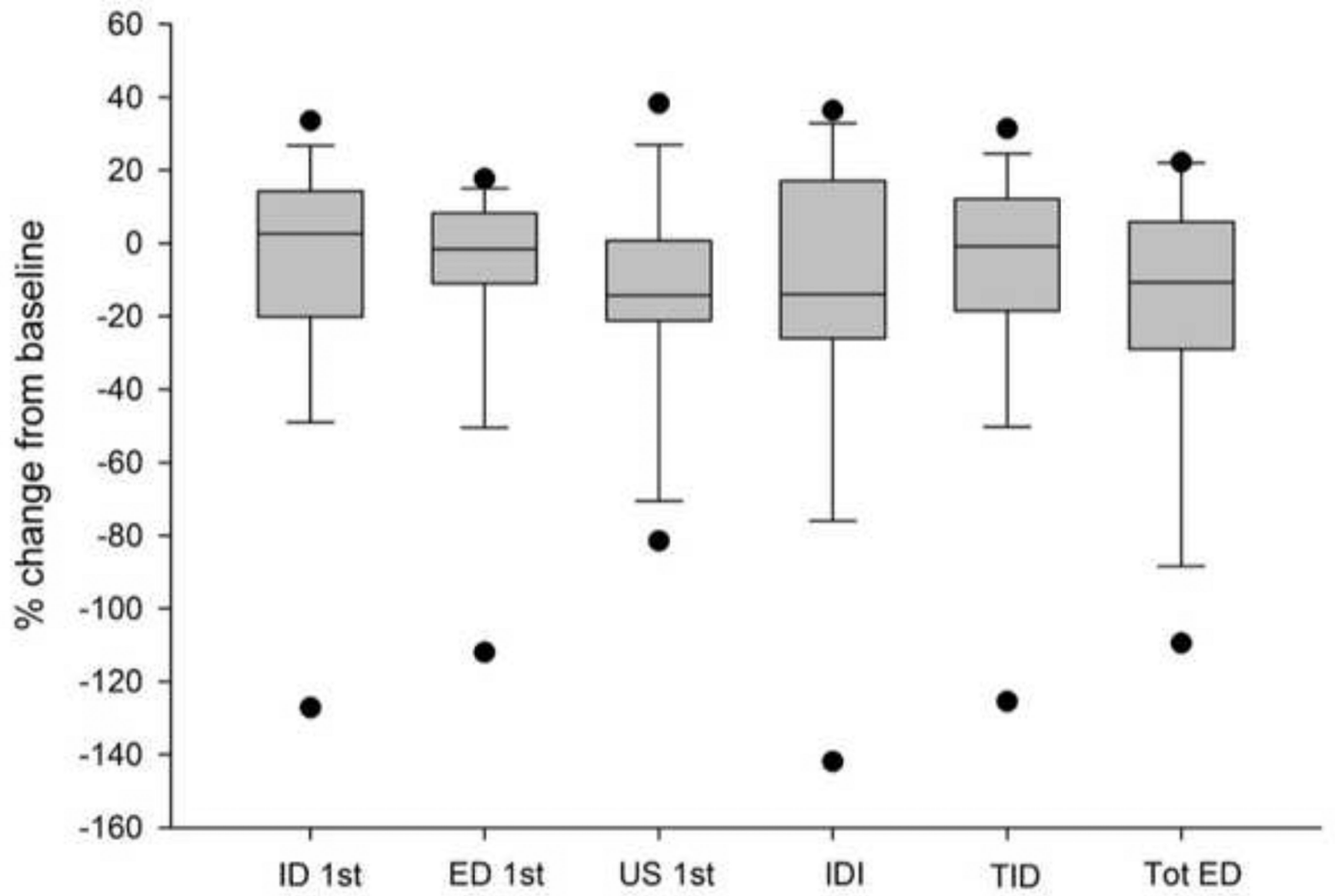

Figure 3.

Percent difference of in vivo RPI measures taken 28 days apart, on contralateral limbs, in untreated skeletally mature male rats. Box plots represent the median, $10^{\text {th }}, 25^{\text {th }}, 75^{\text {th }}$ an $90^{\text {th }}$ percentiles, as well as those individual data points outside this range. 
